\title{
RESTAURANTES SELF-SERVICE NO MUNICÍPIO DE CANOINHAS, SANTA CATARINA, BRASIL: QUALIDADE MICROBIOLÓGICA DOS ALIMENTOS SERVIDOS
}

\author{
Daiana Alves Machado* \\ Bruno Guilherme dos Santos* \\ Graciele Viccini Isaka ${ }^{* \star}$
}

RESUMO: Os serviços de alimentação coletiva têm se expandido em todo o mundo, principalmente os restaurantes do tipo self-service. Esses serviços têm se destacado na epidemiologia dos surtos de doenças transmitidas por alimentos. Assim, o objetivo desta pesquisa foi avaliar a qualidade microbiológica de alimentos (macarrão, carne, alface e maionese) em dez restaurantes tipo self-service em Canoinhas, Santa Catarina, Brasil. Utilizou-se um termômetro e foram feitas análises microbiológicas padronizadas. Com relação à temperatura, nenhuma amostra da cadeia fria encontrava-se em temperatura adequada. Já nos alimentos da cadeia quente, apenas duas amostras de carnes estavam na temperatura correta. Das dez amostras de carnes analisadas, apenas três apresentavam-se propícias para o consumo humano. Das dez amostras de macarrão analisadas, seis encontravam-se aptas para o consumo humano. Todas as amostras de alface e maionese encontravam-se impróprias para o consumo humano. Concluiu-se que pelos altos índices de contaminações encontrados nos alimentos analisados, fica evidente a falta de boas práticas de fabricação nos estabelecimentos participantes. Vale ressaltar que o binômio tempo e temperatura também contribuíram para que ocorressem contaminações.

PALAVRAS-CHAVE: Alimentação coletiva; Doença transmitida por alimento; Segurança alimentar.

\section{SELF-SERVICE RESTAURANTS IN CANOINHAS, BRAZIL: MICROBIOLOGICAL QUALITY OF FOOD}

\begin{abstract}
Community food service is a worldwide phenomenon especially in self-service restaurants, even though it has been underscored for epidemiological onsets of food-transmitted diseases. Current survey evaluates the microbiological quality of food (pasta, beef, lettuces and mayonnaise) in ten self-service restaurants in Canoinhas SC Brazil. Thermometer was employed and standard microbiological analyses were performed. No sample from the cold section presented adequate temperature. In the case of hot food, only two beef samples had the proper temperature. Only three out of the ten beef samples were safe for human consumption. Six out of the ten pasta samples were safe for human consumption. Results show that, due to high contamination indexes in the food analyzed, good practices in the preparation of food are lacking. Time and temperature contribute towards food contamination.
\end{abstract}

KEYWORDS: Community food; Food-transmitted disease; Food safety.

\section{INTRODUÇÃO}

A utilização de restaurantes self-service comida pronta, disposta ao consumidor e vendida por peso - aumentou muito nos últimos anos pela crescente tendência para se alimentar fora de casa
(BADARÓ, 2007). Segundo a Associação Brasileira de Refeições Coletivas (ABERC), em 2017 este mercado estima fornecer 16,7 milhões de refeições/ dia (ABERC, 2017). Além disso, a Pesquisa de Orçamentos Familiares (POF) relatou aumento de 7\% na despesa com alimentação fora do domicílio (média

\footnotetext{
Acadêmica no Instituto Federal de Santa Catarina (IFSC) Campus Canoinhas, Brasil.

** Acadêmico no Instituto Federal de Santa Catarina (IFSC) Campus Canoinhas; Técnico em Agroindústria no (IFSC) Campus Canoinhas, Brasil.

*** Doutora em Ciências-Bioquímica, Docente no Instituto Federal de Santa Catarina (IFSC) Campus Canoinhas; Brasil.

E-mail: graciele.viccini@ifsc.edu.br
} 
mensal familiar) no país, entre 2002/2003 (24,1\%) e 2008/2009 (31,1\%), sendo que na região Sul essa despesa foi de 27,7\% (IBGE, 2010). Em Canoinhas, Santa Catarina, Brasil, o setor de serviços, que inclui restaurantes, contribuiu com $48 \%$ do Valor Adicionado Bruto municipal (SEBRAE, 2013).

Dentre os aspectos relativos à crescente demanda por serviços de refeição fora do lar, a qualidade microbiológica dos produtos oferecidos é fundamental, principalmente considerando a amplitude do público atendido (LIMA et al., 2015).

Segundo Paiva (2016), esses serviços têm se destacado na epidemiologia dos surtos de doenças transmitidas por alimentos (DTAs) em diversos países. Os principais problemas são consequências do reaquecimento e refrigeração inadequados e da preparação de alimentos com muita antecedência, aumentando o tempo de espera para produção e/ou distribuição (MENDES et al., 2011; LIMA et al., 2015).

A qualidade de uma refeição é influenciada por inúmeros fatores: a qualidade da matéria-prima, a higienização dos utensílios utilizados, a higiene dos manipuladores envolvidos no processo, monitoramento de parâmetros, como tempo e temperatura, entre outros (BRAGA et al., 2015).

No entanto, as condições de higiene em que esses alimentos são preparados e a temperatura a que ficam submetidos durante a exposição nem sempre são as ideais, podendo desenvolver micro-organismos potencialmente perigosos para a saúde do consumidor (PENEDO et al., 2015).

Rigorosas práticas higiênicas no preparo são fundamentais para garantir um alimento seguro e evitar as DTAs, que são causadas principalmente pela ingestão de micro-organismos viáveis e/ou toxinas em quantidades suficientes para desenvolver patologias (AFIFI; ABUSHELAIBI, 2012).

0 manipulador tem grande importância em todas as etapas do processo de produção de alimentos, pois pode facilitar a disseminação de micro-organismos deteriorantes e/ou patogênicos no ambiente de trabalho (PAIVA, 2016). A higiene pessoal inadequada é um dos fatores que mais contribuem para a ocorrência de DTAs
(ROCHA et al., 2014). Assim, as mãos de manipuladores podem ser cruciais na contaminação cruzada dentro do ambiente de processamento de alimentos (LUES; VAN TONDER, 2007). Deve-se considerar também que 0 controle da temperatura durante o preparo dos alimentos é fundamental para inibir a multiplicação de micro-organismos (SÃO JOSÉ, 2012).

0 objetivo desta pesquisa foi avaliar a qualidade microbiológica de alimentos em restaurantes comerciais tipo self-service em Canoinhas visto que o consumo de alimentos contaminados por microorganismos patogênicos em restaurantes representa um risco à saúde pública.

\section{METODOLOGIA}

Foram avaliadas a temperatura de preparação e a qualidade microbiológica de dois alimentos da cadeia quente (carne e macarrão) e dois alimentos da cadeia fria (maionese e alface), em dez restaurantes tipo selfservice na região Central do município de Canoinhas, Santa Catarina, Brasil, entre março e maio de 2013. Esses restaurantes representam $50 \%$ desta tipologia, e aceitaram participar deste projeto de pesquisa.

As coletas das amostras foram realizadas após a montagem dos balcões de distribuição, antes do início do serviço. Inicialmente, mediu-se a temperatura dos alimentos expostos nos balcões com termômetro digital infravermelho com mira laser, com faixa de temperatura entre $-32^{\circ} \mathrm{C} \mathrm{a}+380^{\circ} \mathrm{C}$.

Após, foram coletados, de maneira asséptica, pelo menos $200 \mathrm{~g}$ de cada amostra utilizando utensílios e recipientes de vidro estéreis. As amostras foram transportadas ao Laboratório de Microbiologia do Instituto Federal de Santa Catarina - Campus Canoinhas, em caixas isotérmicas com refrigeração. Foram pesados $25 \mathrm{~g}$ de cada amostra, homogeneizados em $225 \mathrm{~mL}$ de água peptonada, para obter a diluição $10^{-1}$. A partir desta, foram feitas, em tubos de ensaio com $9 \mathrm{~mL}$ de água peptonada, diluições decimais sucessivas até $10^{-5}$.

As metodologias foram executadas de acordo com o manual de métodos de análises microbiológicas de alimentos e água (SILVA et al., 2010). 


\subsection{BACTÉRIAS AERÓBIAS MESÓFILAS}

A partir das diluições, alíquotas de $0,1 \mathrm{~mL}$ foram inoculadas em Ágar Padrão para Contagem (PCA) e incubadas a $35^{\circ} \mathrm{C}$ por 48 horas.

\subsection{COLIFORMES TOTAIS E TERMOTOLERANTES}

A determinação do Número Mais Provável (NMP/g) de coliformes totais e termotolerantes foi feita pela técnica de tubos múltiplos. Foi inoculado $1 \mathrm{~mL}$ de cada diluição em três tubos com Caldo Lauril Sulfato Triptose (LST) para teste presuntivo, e incubados a $35^{\circ} \mathrm{C}$ por 48 horas. Para cada tubo de caldo LST positivo foi transferida uma alçada para um tubo com Caldo Verde Brilhante e Bile $2 \%$ (VBB), para determinar a presença de coliformes totais, e uma alçada para um tubo com Caldo EC, para determinar a presença de coliformes termotolerantes. Os tubos de VBB foram incubados a $35^{\circ} \mathrm{C}$ por 48 horas e os tubos de EC foram incubados a $45^{\circ} \mathrm{C}$ por 48 horas.

\subsection{PESQUISA DE SALMONELLA SPP.}

Foram transferidos $25 \mathrm{~g}$ da amostra para 225 $\mathrm{mL}$ de caldo de pré-enriquecimento, que foi incubado a $35^{\circ} \mathrm{C}$ por 24 horas. Após, foi transferido $0,1 \mathrm{~mL}$ para um tubo com Caldo Rappaport Vassiliadis, incubado a $42^{\circ} \mathrm{C}$ por 24 horas, e $1 \mathrm{~mL}$ para um tubo com Caldo Tetrationato Muller, incubado a $35^{\circ} \mathrm{C}$ por 24 horas. Depois, a partir de cada tubo foram feitas estriagens por esgotamento, utilizando alça de semeadura, em placas contendo os meios de cultivo Ágar Xilose Lisina Desoxicolato e Ágar Bismuto Sulfito, incubadas a $37^{\circ} \mathrm{C}$ por 48 horas.

\subsection{STAPHYLOCOCCUS COAGULASE POSITIVA}

Após as diluições, alíquotas de $0,1 \mathrm{~mL}$ foram inoculadas em meio Ágar Baird Parker e incubadas a $35^{\circ} \mathrm{C}$ por 48 horas.

\subsection{CONTAGEM DE BACILLUS CEREUS}

A partir das diluições, alíquotas de $0,1 \mathrm{~mL}$ foram semeadas em meio Ágar Manitol Gema de Ovo Polimixina, incubadas a $30^{\circ} \mathrm{C}$ por 24 horas.

\subsection{ANÁLISES DOS RESULTADOS}

Os resultados obtidos nas análises microbiológicas foram comparados com a resolução n¹2/2001 da Agência Nacional de Vigilância Sanitária (ANVISA) (BRASIL, 2001) e com 0 artigo científico de Alves e Ueno (2010). Para coliformes totais foram adotados os mesmos valores limites estabelecidos pela legislação para coliformes termotolerantes.

\section{RESULTADOS E DISCUSSÃO}

\subsection{TEMPERATURA DOS ALIMENTOS}

Durante a coleta das amostras foi realizada a avaliação da temperatura dos alimentos da cadeia fria e da cadeia quente, visto que o emprego de temperaturas inadequadas durante a disposição dos alimentos em restaurantes favorece 0 desenvolvimento microbiano e, consequentemente, a contaminação dos alimentos.

A resolução $n^{\circ} 216 / 2004$ estabelece que os alimentos devem ser submetidos, durante toda cocção, à temperatura de, no mínimo, $70^{\circ} \mathrm{C}$ em todo 0 seu centro geométrico para garantir a segurança ao consumo de tais alimentos (BRASIL, 2004). Além disso, o binômio tempo/temperatura a que as preparações são expostas durante a etapa de distribuição das refeições também é muito importante para a segurança dos alimentos. Para conservação a quente, os alimentos devem ser submetidos a temperaturas superiores a $60^{\circ} \mathrm{C}$ por no máximo 6 horas (BRASIL, 2013b; PENED0 et al., 2015). A temperatura ideal para exposição de alimentos da cadeia fria é de $6^{\circ} \mathrm{C}$, não devendo ultrapassar os $10^{\circ} \mathrm{C}$ (SEREJ0 et al., 2010).

Nos restaurantes avaliados nessa pesquisa, nenhuma amostra de alimento da cadeia fria estava 
em temperatura adequada, sendo que as temperaturas variaram entre $11,4^{\circ} \mathrm{C}$ e $27,4^{\circ} \mathrm{C}$. Já nos alimentos da cadeia quente, apenas duas amostras de carne estavam expostas na temperatura correta, sendo que as temperaturas mensuradas variaram entre $34,7^{\circ} \mathrm{C}$ e $82^{\circ} \mathrm{C}$, como podem ser observadas na Figura 1.

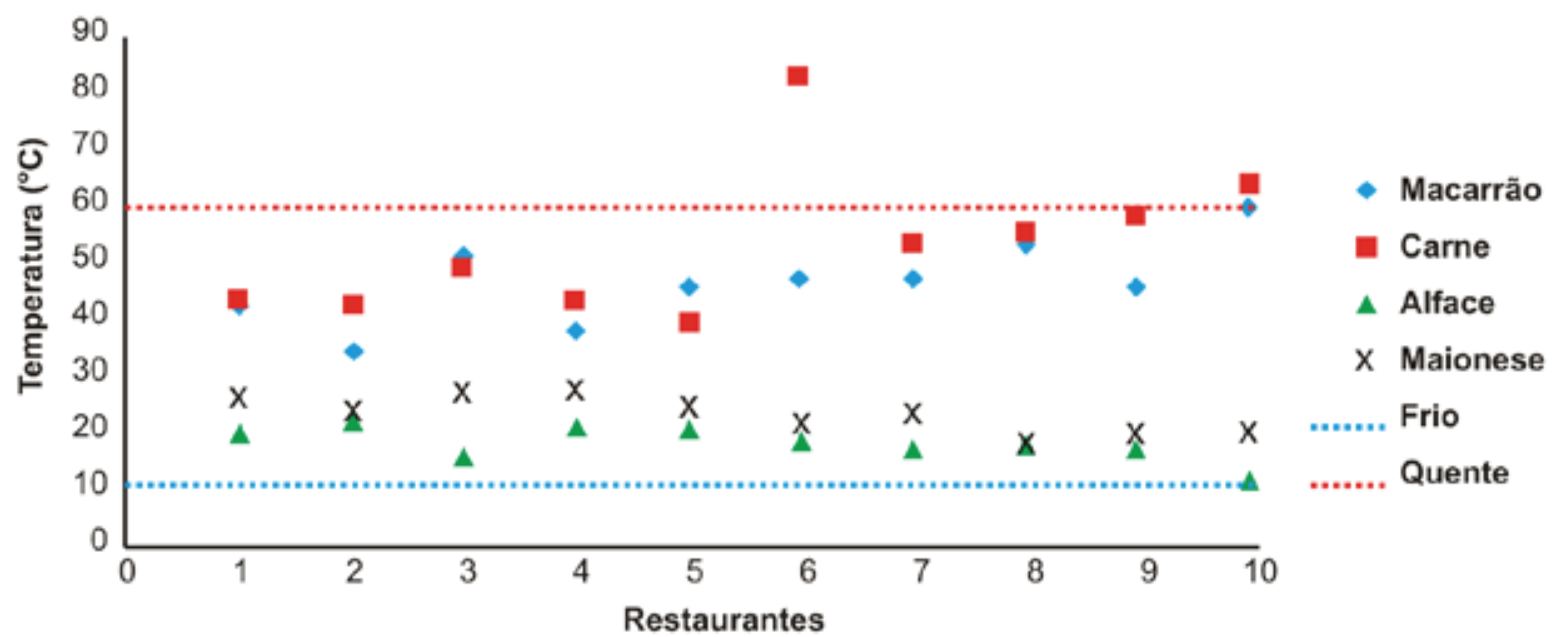

Figura 1. Temperatura dos alimentos da cadeia fria e da cadeia quente, coletados em dez restaurantes tipo self-service em Canoinhas - SC

Resultados semelhantes foram encontrados por Penedo et al. (2015), que avaliaram as temperaturas do prato principal, da guarnição, do acompanhamento e da salada durante o preparo e distribuição em dez restaurantes comerciais do tipo self-service de Belo Horizonte, Minas Gerais, Brasil. Os resultados indicaram que $60 \%$ dos restaurantes analisados apresentaram temperaturas impróprias entre as preparações. A variação da temperatura dos pratos quentes foi de $45^{\circ} \mathrm{C}$ a $91^{\circ} \mathrm{C}$ e dos pratos frios ficou entre $9^{\circ} \mathrm{C} \mathrm{e} 24^{\circ} \mathrm{C}$.

Oliveira et al. (2012) também constataram altos níveis de inadequação quando avaliaram a temperatura de alimentos servidos em 76 restaurantes de Belo Horizonte. Apenas 1,3\% das amostras de feijão e de massas encontrava-se em temperatura ideal, seguido por $2,7 \%$ das amostras de frituras, $6,7 \%$ das amostras de carne de aves e $8,1 \%$ das amostras de carne bovina. Já em relação aos alimentos da cadeia fria, apenas $1,3 \%$ das amostras de tomate estavam em temperaturas ideais.

Santos e Bassi (2015) realizaram a avaliação da temperatura de alimentos servidos em oito unidades de alimentação e nutrição em São Paulo, Brasil. As temperaturas dos pratos principais e sobremesas estavam dentro dos limites estabelecidos pela legislação. Entretanto, as preparações como 0 arroz/feijão, as guarnições e as saladas apresentaram índices de inadequação de $87,5 \%, 62,5 \%$ e 12,5\%, respectivamente.

Em estudo realizado por Alves e Ueno (2010), sobre a temperatura de exposição dos alimentos, constataram que apenas $22 \%$ dos alimentos da cadeia quente encontravam-se na temperatura correta e apenas $3,7 \%$ dos alimentos da cadeia fria apresentavam-se em temperaturas ideais.

De maneira semelhante, Alves e Mesquita (2010) relataram a adequação de apenas $25 \%$ dos alimentos em relação à temperatura de exposição em uma unidade de alimentação comercial do município de Santa Maria, Rio Grande do Sul, Brasil.

0 monitoramento das temperaturas dos alimentos deve ser constante, a fim de minimizar riscos de contaminação e crescimento microbiológico para evitar danos à saúde dos clientes (SANTOS; BASSI, 2015). 
3.2 ANÁLISES MICROBIOLÓGICAS

0 Ministério da Saúde estabelece, na resolução $n^{\circ} 12$ (BRASIL, 2001), as seguintes pesquisas microbiológicas de interesse sanitário para pratos prontos para 0 consumo (alimentos prontos de cozinhas, restaurantes e similares): quantificação de bactérias aeróbias mesófilas, de coliformes totais e termotolerantes, de Bacillus cereus e pesquisa de Staphylococcus coagulase positiva e de Salmonella spp.
Das dez amostras de carnes analisadas, apenas três apresentaram-se propícias para 0 consumo humano. As demais amostras apresentaram contaminação por Salmonella spp. (5/10), coliformes totais (1/10), coliformes termotolerantes (2/10) e por $B$. cereus (2/10). Nenhuma amostra apresentou resultado em desacordo com a legislação para $S$. aureus e as contagens de bactérias aeróbias mesófilas também estavam de acordo com o valor de referência utilizado, como pode ser observado na Tabela 1.

Tabela 1. Resultados das análises microbiológicas das amostras de carne coletadas em dez restaurantes tipo self-service em Canoinhas (SC)

\begin{tabular}{|c|c|c|c|c|c|c|c|c|c|c|}
\hline \multicolumn{11}{|c|}{ Alimento: Carne } \\
\hline \multicolumn{11}{|c|}{ Restaurantes } \\
\hline Análises & A & B & C & D & E & $\mathbf{F}$ & G & H & I & J \\
\hline $\begin{array}{l}\text { Bactérias } \\
\text { aeróbias } \\
\text { mesófilas }\end{array}$ & $\begin{array}{c}<1,0 \times 10^{2} \\
\text { UFC/g } \\
\text { (est.) }\end{array}$ & $\begin{array}{c}1,2 \times 10^{2} \\
\text { UFC/g } \\
\text { (est.) }\end{array}$ & $\begin{array}{c}6,0 \times 10^{2} \\
\text { UFC/g } \\
\text { (est.) }\end{array}$ & $\begin{array}{c}6,0 \times 10^{2} \\
\text { UFC/g } \\
\text { (est.) }\end{array}$ & $\begin{array}{c}2,6 \times 10^{2} \\
\text { UFC/g }\end{array}$ & $\begin{array}{c}1,0 \times 10^{2} \\
\text { UFC/g } \\
\text { (est.) }\end{array}$ & $\begin{array}{c}3,1 \times 10^{2} \\
\text { UFC/g }\end{array}$ & $\begin{array}{c}<1,0 \times 10^{2} \\
\text { UFC/g } \\
\text { (est.) }\end{array}$ & $\begin{array}{c}2,4 \times 10^{2} \\
\text { UFC/g }\end{array}$ & $\begin{array}{c}7,9 \times 10^{2} \\
\text { UFC/g }\end{array}$ \\
\hline $\begin{array}{l}\text { Estafilococus } \\
\text { coagulas e } \\
\text { positiva }\end{array}$ & $\begin{array}{c}<1,0 \times 10^{2} \\
\text { UFC/g } \\
\text { (est.) }\end{array}$ & $\begin{array}{c}1,0 \times 10^{2} \\
\text { UFC/g } \\
\text { (est.) }\end{array}$ & $\begin{array}{c}1,0 \times 10^{2} \\
\text { UFC/g } \\
\text { (est.) }\end{array}$ & $\begin{array}{c}<1,0 \times 10^{2} \\
\text { UFC/g } \\
\text { (est.) }\end{array}$ & $\begin{array}{c}<1,0 \times 10^{2} \\
\text { UFC/g } \\
\text { (est.) }\end{array}$ & $\begin{array}{c}<1,0 \times 10^{2} \\
\text { UFC/g } \\
\text { (est.) }\end{array}$ & $\begin{array}{c}<1,0 \times 10^{2} \\
\text { UFC/g } \\
\text { (est.) }\end{array}$ & $\begin{array}{c}<1,0 \times 10^{2} \\
\text { UFC/g } \\
\text { (est.) }\end{array}$ & $\begin{array}{c}<1,0 \times 10^{2} \\
\text { UFC/g } \\
\text { (est.) }\end{array}$ & $\begin{array}{c}<1,0 \times 10^{2} \\
\text { UFC/g } \\
\text { (est.) }\end{array}$ \\
\hline Bacillus cereus & $\begin{array}{c}<1,0 \times 10^{2} \\
\text { UFC/g } \\
\text { (est.) }\end{array}$ & $\begin{array}{l}7,0 \times 10^{2} \\
\text { UFC/g } \\
\text { (est.) }\end{array}$ & $\begin{array}{c}1,0 \times 10^{2} \\
\text { UFC/g } \\
\text { (est.) }\end{array}$ & $\begin{array}{c}<1,0 \times 10^{2} \\
\text { UFC/g } \\
\text { (est.) }\end{array}$ & $\begin{array}{c}<1,0 \times 10^{2} \\
\text { UFC/g } \\
\text { (est.) }\end{array}$ & $\begin{array}{c}<1,0 \times 10^{2} \\
\text { UFC/g } \\
\text { (est.) }\end{array}$ & $\begin{array}{c}<1,0 \times 10^{2} \\
\text { UFC/g } \\
\text { (est.) }\end{array}$ & $\begin{array}{c}<1,0 \times 10^{2} \\
\text { UFC/g } \\
\text { (est.) }\end{array}$ & $\begin{array}{l}8,2 \times 10^{2} \\
\text { UFC/g* }\end{array}$ & $\begin{array}{c}4,0 \times 10^{2} \\
\text { UFC/g } \\
\text { (est.)* }\end{array}$ \\
\hline Salmonella spp. & Ausência & $\begin{array}{l}\text { Possível } \\
\text { presença } \\
\star\end{array}$ & $\begin{array}{c}\text { Possível } \\
\text { presença } \\
\star\end{array}$ & Ausência & Ausência & Ausência & $\begin{array}{c}\text { Possível } \\
\text { presença } \\
\star\end{array}$ & $\begin{array}{c}\text { Possível } \\
\text { presença } \\
\star\end{array}$ & Ausência & Ausência \\
\hline $\begin{array}{l}\text { Coliformes } \\
\text { totais }\end{array}$ & $\begin{array}{c}<3,0 \\
\mathrm{NMP} / \mathrm{g}\end{array}$ & $\begin{array}{c}<3,0 \\
\mathrm{NMP} / \mathrm{g}\end{array}$ & $\begin{array}{c}<3,0 \\
\mathrm{NMP} / \mathrm{g}\end{array}$ & $\begin{array}{l}3,6 \times 10^{1} \\
\text { NMP/g* }\end{array}$ & $\begin{array}{c}<3,0 \\
\mathrm{NMP} / \mathrm{g}\end{array}$ & $\begin{array}{c}<3,0 \\
\mathrm{NMP} / \mathrm{g}\end{array}$ & $\begin{array}{l}2,3 \times 10^{1} \\
\text { NMP/g* }\end{array}$ & $\begin{array}{c}<3,0 \\
\mathrm{NMP} / \mathrm{g}\end{array}$ & $\begin{array}{c}<3,0 \\
\mathrm{NMP} / \mathrm{g}\end{array}$ & $\begin{array}{l}<3,0 \\
\mathrm{NMP} / \mathrm{g}\end{array}$ \\
\hline $\begin{array}{l}\text { Coliformes } \\
\text { termotolerantes }\end{array}$ & $\begin{array}{l}<3,0 \\
\mathrm{NMP} / \mathrm{g}\end{array}$ & $\begin{array}{l}<3,0 \\
\mathrm{NMP} / \mathrm{g}\end{array}$ & $\begin{array}{l}<3,0 \\
\mathrm{NMP} / \mathrm{g}\end{array}$ & $\begin{array}{l}<3,0 \\
\mathrm{NMP} / \mathrm{g}\end{array}$ & $\begin{array}{l}2,3 \times 10^{1} \\
\mathrm{NMP} / \mathrm{g}^{\star}\end{array}$ & $\begin{array}{l}<3,0 \\
\mathrm{NMP} / \mathrm{g}\end{array}$ & $\begin{array}{l}<3,0 \\
\mathrm{NMP} / \mathrm{g}\end{array}$ & $\begin{array}{l}9,2 \times 10^{1} \\
\mathrm{NMP} / \mathrm{g}^{*}\end{array}$ & $\begin{array}{l}<3,0 \\
\mathrm{NMP} / \mathrm{g}\end{array}$ & $\begin{array}{l}3,6 \times 10^{1} \\
\mathrm{NMP} / \mathrm{g}^{\star}\end{array}$ \\
\hline
\end{tabular}

* Amostra imprópria para consumo humano segundo padrão microbiológico estabelecido pela RDC $n^{0} 12$ da ANVISA (BRASIL, 2001).

${ }^{* *}$ Amostra imprópria para consumo humano segundo limite para Coliformes Termotolerantes da RDC $n^{0} 12$ da ANVISA (BRASIL, 2001).

*** Amostra imprópria para consumo humano segundo critérios de avaliação de Alves, Ueno (2010). 
Em estudo realizado por Alves e Ueno (2010), nenhuma amostra de carne teve contaminação por Salmonella spp. e apenas uma apresentou resultado que ultrapassou os limites para $S$. aureus. Da mesma forma, Dolinger et al. (2010) realizaram análises microbiológicas em 23 amostras de carnes provenientes de restaurantes self-service de Itumbiara, Goiás, Brasil, e apenas duas amostras excederam os limites microbiológicos para coliformes termotolerantes.
Badaró (2007) analisou 20 amostras de carne provenientes de restaurantes self-service de Ipatinga, Minas Gerais, Brasil, e 25\% delas encontravam-se impróprias para o consumo, apresentando níveis de contaminação microbiológica acima do permitido pela legislação vigente.

Tabela 2. Resultados das análises microbiológicas das amostras de macarrão coletadas em dez restaurantes tipo selfservice em Canoinhas - SC

Alimento: Macarrão

Restaurantes

\begin{tabular}{|c|c|c|c|c|c|c|c|c|c|c|}
\hline Análises & A & B & C & D & E & $\mathbf{F}$ & G & H & I & $\mathbf{J}$ \\
\hline $\begin{array}{l}\text { Bactérias } \\
\text { aeróbias } \\
\text { mesófilas }\end{array}$ & $\begin{array}{c}<1,0 \times 10^{2} \\
\text { UFC/g } \\
\text { (est.) }\end{array}$ & $\begin{array}{c}1,5 \times 10^{2} \\
\text { UFC/g } \\
\text { (est.) }\end{array}$ & $\begin{array}{c}6,0 \times 10^{2} \\
\text { UFC/g } \\
\text { (est.) }\end{array}$ & $\begin{array}{c}1,0 \times 10^{2} \\
\text { UFC/g } \\
\text { (est.) }\end{array}$ & $\begin{array}{l}9,2 \times 10^{2} \\
\text { UFC/g }\end{array}$ & $\begin{array}{c}1,0 \times 10^{2} \\
\text { UFC/g } \\
\text { (est.) }\end{array}$ & $\begin{array}{c}9,4 \times 10^{4} \\
\text { UFC/g }\end{array}$ & $\begin{array}{c}1,6 \times 10^{7} \\
\text { UFC/g } \\
\text { (est.) }\end{array}$ & $\begin{array}{c}<1,0 \times 10^{2} \\
\text { UFC/g } \\
\text { (est.) }\end{array}$ & $\begin{array}{c}<1,0 \times 10^{2} \\
\text { UFC/g } \\
\text { (est.) }\end{array}$ \\
\hline $\begin{array}{l}\text { Estafilococus } \\
\text { coagulas e } \\
\text { positiva }\end{array}$ & $\begin{array}{c}<1,0 \times 10^{2} \\
\text { UFC/g } \\
\text { (est.) }\end{array}$ & $\begin{array}{l}5,0 \times 10^{2} \\
\text { UFC/g } \\
\text { (est.)* }\end{array}$ & $\begin{array}{l}3,0 \times 10^{4} \\
\text { UFC/g } \\
\text { (est.) }^{*}\end{array}$ & $\begin{array}{c}<1,0 \times 10^{2} \\
\text { UFC/g } \\
\text { (est.) }\end{array}$ & $\begin{array}{c}<1,0 \times 10^{2} \\
\text { UFC/g } \\
\text { (est.) }\end{array}$ & $\begin{array}{c}<1,0 \times 10^{2} \\
\text { UFC/g } \\
\text { (est.) }\end{array}$ & $\begin{array}{c}<1,0 \times 10^{2} \\
\text { UFC/g } \\
\text { (est.) }\end{array}$ & $\begin{array}{c}1,3 \times 10^{2} \\
\text { UFC/g }\end{array}$ & $\begin{array}{c}<1,0 \times 10^{2} \\
\text { UFC/g } \\
\text { (est.) }\end{array}$ & $\begin{array}{c}<1,0 \times 10^{2} \\
\text { UFC/g } \\
\text { (est.) }\end{array}$ \\
\hline Bacillus cereus & $\begin{array}{c}<1,0 \times 10^{2} \\
\text { UFC/g } \\
\text { (est.) }\end{array}$ & $\begin{array}{c}4,0 \times 10^{2} \\
\text { UFC/g } \\
\text { (est.) }\end{array}$ & $\begin{array}{l}\text { 1,0x104 } \\
\text { UFC/g } \\
\text { (est.)* }^{*}\end{array}$ & $\begin{array}{c}<1,0 \times 10^{2} \\
\text { UFC/g } \\
\text { (est.) }\end{array}$ & $\begin{array}{c}<1,0 \times 10^{2} \\
\text { UFC/g } \\
\text { (est.) }\end{array}$ & $\begin{array}{c}<1,0 \times 10^{2} \\
\text { UFC/g } \\
\text { (est.) }\end{array}$ & $\begin{array}{c}<1,0 \times 10^{2} \\
\text { UFC/g } \\
\text { (est.) }\end{array}$ & $\begin{array}{l}4,0 \times 10^{3} \\
\text { UFC/g } /{ }^{*}\end{array}$ & $\begin{array}{c}5,0 \times 10^{2} \\
\text { UFC/g } \\
\text { (est.) }\end{array}$ & $\begin{array}{c}9,0 \times 10^{2} \\
\text { UFC/g } \\
\text { (est.)* }^{*}\end{array}$ \\
\hline Salmonella spp. & Ausência & $\begin{array}{c}\text { Possível } \\
\text { presença } \\
\text { * }\end{array}$ & $\begin{array}{c}\text { Possível } \\
\text { presença } \\
\text { * }\end{array}$ & Ausência & Ausência & Ausência & $\begin{array}{c}\text { Possível } \\
\text { presença } \\
\star\end{array}$ & $\begin{array}{l}\text { Possível } \\
\text { presença } \\
\star\end{array}$ & Ausência & Ausência \\
\hline $\begin{array}{l}\text { Coliformes } \\
\text { totais }\end{array}$ & $\begin{array}{c}<3,0 \\
\mathrm{NMP} / \mathrm{g}\end{array}$ & $\begin{array}{c}<3,0 \\
\mathrm{NMP} / \mathrm{g}\end{array}$ & $\begin{array}{c}<3,0 \\
\mathrm{NMP} / \mathrm{g}\end{array}$ & $\begin{array}{c}<3,0 \\
\mathrm{NMP} / \mathrm{g}\end{array}$ & $\begin{array}{c}<3,0 \\
\mathrm{NMP} / \mathrm{g}\end{array}$ & $\begin{array}{c}<3,0 \\
\mathrm{NMP} / \mathrm{g}\end{array}$ & $\begin{array}{l}3,6 \times 10^{1} \\
\mathrm{NMP} / \mathrm{g}^{*}\end{array}$ & $\begin{array}{l}2,3 \times 10^{1} \\
\text { NMP/g }\end{array}$ & $\begin{array}{c}<3,0 \\
\mathrm{NMP} / \mathrm{g}\end{array}$ & $\begin{array}{c}<3,0 \\
\mathrm{NMP} / \mathrm{g}\end{array}$ \\
\hline $\begin{array}{l}\text { Coliformes } \\
\text { termotolerantes }\end{array}$ & $\begin{array}{l}<3,0 \\
\mathrm{NMP} / \mathrm{g}\end{array}$ & $\begin{array}{l}<3,0 \\
\mathrm{NMP} / \mathrm{g}\end{array}$ & $\begin{array}{l}<3,0 \\
\mathrm{NMP} / \mathrm{g}\end{array}$ & $\begin{array}{l}<3,0 \\
\mathrm{NMP} / \mathrm{g}\end{array}$ & $\begin{array}{l}2,3 \times 10^{1} \\
\mathrm{NMP} / \mathrm{g}^{*}\end{array}$ & $\begin{array}{l}<3,0 \\
\mathrm{NMP} / \mathrm{g}\end{array}$ & $\begin{array}{l}<3,0 \\
\mathrm{NMP} / \mathrm{g}\end{array}$ & $\begin{array}{l}9,2 \times 10^{1} \\
\text { NMP/g* }\end{array}$ & $\begin{array}{l}<3,0 \\
\mathrm{NMP} / \mathrm{g}\end{array}$ & $\begin{array}{l}<3,0 \\
\mathrm{NMP} / \mathrm{g}\end{array}$ \\
\hline
\end{tabular}

${ }^{*}$ Amostra imprópria para consumo humano segundo padrão microbiológico estabelecido pela RDC $n^{\circ} 12$ da ANVISA (BRASIL, 2001).

${ }^{* *}$ Amostra imprópria para consumo humano segundo limite para Coliformes Termotolerantes da RDC n² 12 da ANVISA (BRASIL, 2001).

*** Amostra imprópria para consumo humano segundo critérios de avaliação de Alves, Ueno (2010). 
Como pode ser observado na Tabela 2, das dez amostras de macarrão analisadas, seis encontravamse aptas para o consumo humano. Nas demais amostras foi constatada contaminação por $S$. aureus (3/10), $B$. cereus (2/10), Salmonella spp. (4/10), coliformes totais $(2 / 10)$ e coliformes termotolerantes (1/10). Quanto à contagem de bactérias aeróbias mesófilas, apenas uma amostra excedeu o limite microbiológico de acordo com o valor de referência utilizado.

Resultados contrários foram relatados por Resta e Oliveira (2013) que avaliaram a presença de $S$. aureus em 50 amostras de macarrão e não encontraram nenhuma amostra excedendo o limite microbiológico determinado pela resolução $n^{\circ} 12$ (BRASIL, 2001). Da mesma forma, Dolinger et al. (2010) analisaram 23 amostras de macarronada provenientes de restaurantes de Itumbiara e constataram que apenas duas amostras estavam impróprias para 0 consumo, apresentando contaminação por coliformes termotolerantes.

Todasasamostras dealface estavam impróprias para o consumo humano, pois ultrapassaram os limites da resolução $n^{\circ} 12$ (BRASIL, 2001) para coliformes termotolerantes (8/10), como pode ser observado na Tabela 3. Além disso, apresentaram possível presença de Salmonella spp. em $100 \%$ das amostras. Foram encontrados também valores elevados de coliformes totais $(9 / 10)$ e de bactérias aeróbias mesófilas $(3 / 10)$, de acordo com os valores de referência utilizados.

Tabela 3. Resultados das análises microbiológicas das amostras de alface coletadas em dez restaurantes tipo self-service em Canoinhas - SC

\begin{tabular}{|c|c|c|c|c|c|c|c|c|c|c|}
\hline \multicolumn{11}{|c|}{ Alimento: Alface } \\
\hline \multicolumn{11}{|c|}{ Restaurantes } \\
\hline Análises & A & B & C & D & $\mathbf{E}$ & $\mathbf{F}$ & $\mathbf{G}$ & H & I & $\mathbf{J}$ \\
\hline $\begin{array}{l}\text { Bactérias } \\
\text { aeróbias } \\
\text { mesófilas }\end{array}$ & $\begin{array}{l}1,6 \times 10^{5} \\
\text { UFC/g* }\end{array}$ & $\begin{array}{l}6,0 \times 10^{6} \\
\text { UFC/g* }\end{array}$ & $\begin{array}{l}5,7 \times 10^{5} \\
\text { UFC/g }\end{array}$ & $\begin{array}{l}1,5 \times 10^{5} \\
\text { UFC/g }\end{array}$ & $\begin{array}{l}1,5 \times 10^{4} \\
\text { UFC/g }\end{array}$ & $\begin{array}{l}9,2 \times 10^{4} \\
\text { UFC/g }\end{array}$ & $\begin{array}{l}7,8 \times 10^{4} \\
\text { UFC/g }\end{array}$ & $\begin{array}{l}5,8 \times 10^{5} \\
\text { UFC/g* }\end{array}$ & $\begin{array}{c}2,5 \times 10^{2} \\
\text { UFC/g }\end{array}$ & $\begin{array}{l}1,4 \times 10^{5} \\
\text { UFC/g }\end{array}$ \\
\hline Salmonella spp. & $\begin{array}{c}\text { Possível } \\
\text { presença } \\
\star\end{array}$ & $\begin{array}{c}\text { Possível } \\
\text { presença } \\
\star\end{array}$ & $\begin{array}{c}\text { Possível } \\
\text { presença } \\
\star\end{array}$ & $\begin{array}{c}\text { Possível } \\
\text { presença } \\
\star\end{array}$ & $\begin{array}{l}\text { Possível } \\
\text { presença } \\
\star\end{array}$ & $\begin{array}{l}\text { Possível } \\
\text { presença } \\
\text { * }\end{array}$ & $\begin{array}{c}\text { Possível } \\
\text { presença } \\
\star\end{array}$ & $\begin{array}{l}\text { Possível } \\
\text { presença } \\
\star\end{array}$ & $\begin{array}{c}\text { Possível } \\
\text { presença } \\
\star\end{array}$ & $\begin{array}{c}\text { Possível } \\
\text { presença } \\
\star\end{array}$ \\
\hline $\begin{array}{l}\text { Coliformes } \\
\text { totais }\end{array}$ & $\begin{array}{c}>1,1 \times 10^{3} \\
\mathrm{NMP} / \mathrm{g}^{\star}\end{array}$ & $\begin{array}{c}>1,1 \times 10^{3} \\
\mathrm{NMP} / \mathrm{g}^{*}\end{array}$ & $\begin{array}{l}7,5 \times 10^{2} \\
\text { NMP/g }\end{array}$ & $\begin{array}{l}4,3 \times 10^{2} \\
\mathrm{NMP} / \mathrm{g}^{*}\end{array}$ & $\begin{array}{l}9,3 \times 10^{2} \\
\text { NMP } / g^{*}\end{array}$ & $\begin{array}{l}2,3 \times 10^{2} \\
\mathrm{NMP} / \mathrm{g}^{\star}\end{array}$ & $\begin{array}{c}2,4 \times 10^{1} \\
\mathrm{NMP} / \mathrm{g}\end{array}$ & $\begin{array}{l}2,5 \times 10^{3} \\
\mathrm{NMP} / \mathrm{g}^{\star}\end{array}$ & $\begin{array}{l}1,1 \times 10^{2} \\
\mathrm{NMP} / \mathrm{g}^{*}\end{array}$ & $\begin{array}{l}1,1 \times 10^{3} \\
\mathrm{NMP} / \mathrm{g}^{*}\end{array}$ \\
\hline $\begin{array}{c}\text { Coliformes } \\
\text { termotolerantes }\end{array}$ & $\begin{array}{c}4,3 \times 10^{1} \\
\mathrm{NMP} / 9\end{array}$ & $\begin{array}{c}>1,1 \times 10^{5} \\
\mathrm{NMP} / \mathrm{g}^{\star}\end{array}$ & $\begin{array}{l}2,3 \times 10^{2} \\
\mathrm{NMP} / \mathrm{g}^{\star}\end{array}$ & $\begin{array}{l}9,3 \times 10^{1} \\
\mathrm{NMP} / \mathrm{g}^{\star}\end{array}$ & $\begin{array}{l}1,2 \times 10^{2} \\
\mathrm{NMP} / \mathrm{g}^{\star}\end{array}$ & $\begin{array}{l}2,3 \times 10^{2} \\
\mathrm{NMP} / \mathrm{g}^{\star}\end{array}$ & $\begin{array}{l}<3,0 \\
\mathrm{NMP} / \mathrm{g}\end{array}$ & $\begin{array}{l}9,2 \times 10^{1} \\
\mathrm{NMP} / \mathrm{g}^{\star}\end{array}$ & $\begin{array}{l}2,3 \times 10^{1} \\
\mathrm{NMP} / \mathrm{g}^{\star}\end{array}$ & $\begin{array}{l}2,9 \times 10^{2} \\
\mathrm{NMP} / \mathrm{g}^{\star}\end{array}$ \\
\hline
\end{tabular}

* Amostra imprópria para consumo humano segundo padrão microbiológico estabelecido pela RDC $n^{0} 12$ da ANVISA (BRASIL, 2001).

${ }^{* *}$ Amostra imprópria para consumo humano segundo limite para Coliformes Termotolerantes da RDC $n^{0} 12$ da ANVISA (BRASIL, 2001).

*** Amostra imprópria para consumo humano segundo critérios de avaliação de Alves, Ueno (2010). 
Resultados semelhantes a estes foram relatados por Rocha et al. (2014), que realizaram a análise microbiológica de nove amostras de saladas cruas servidas em restaurantes de Teresina, Piauí, Brasil. 0s resultados indicaram a presença de coliformes totais e termotolerantes em $100 \%$ das amostras, além de contaminação por bactérias aeróbias mesófilas. Houve presença de Salmonella spp. em $11,11 \%$ das saladas cruas.

Da mesma forma, Rodrigues et al. (2012) analisaram a presença de Salmonella spp., coliformes totais e termotolerantes em 30 amostras de alface provenientes de dez restaurantes self-service do município de Natal, Rio Grande do Norte, Brasil. Embora nenhuma amostra tenha apresentado a presença de Salmonella spp., 93,3\% das amostras obtiveram contagens acima do limite permitido pela legislação.

Lima et al. (2015) analisaram dez amostras de saladas e também relataram que $40 \%$ das amostras tiveram resultados superiores ao permitido pela legislação para coliformes totais e termotolerantes.

Ao contrário destes resultados, Calil et al. (2013) analisaram 30 amostras de saladas verdes provenientes de seis restaurantes self-service de São Bernardo do Campo, São Paulo, Brasil, e apenas uma amostra ultrapassou os limites permitidos pela legislação para $S$. aureus, em que foi obtido um valor de $64 \times 10^{2} \mathrm{UFC} / \mathrm{g}$. As demais apresentaram resultados microbiológicos dentro dos limites permitidos pela legislação.

Santa et al. (2011) também apresentaram resultados satisfatórios para as análises de 24 amostras de saladas provenientes de oito restaurantes selfservice de Guarapuava, Paraná, Brasil. Destas, cinco amostras eram de alface, em que foram detectadas contagens elevadas de coliformes totais. Nas demais amostras, os valores encontrados para coliformes termotolerantes e para Salmonella spp. estavam dentro do permitido pela legislação vigente.

Todas as amostras de maionese analisadas neste estudo apresentaram-se impróprias para 0 consumo humano por conterem contaminação por Salmonella spp. (10/10), coliformes totais (10/10), coliformes termotolerantes (7/10), $S$. aureus (5/10), $B$. cereus $(2 / 10)$ e bactérias aeróbias mesófilas $(1 / 10)$, como mostra a Tabela 4.

Tabela 4. Resultados das análises microbiológicas das amostras de maionese coletadas em dez restaurantes tipo selfservice em Canoinhas - SC

Alimento: Maionese

\begin{tabular}{|c|c|c|c|c|c|c|c|c|c|c|}
\hline \multicolumn{11}{|c|}{ Restaurantes } \\
\hline Análises & A & B & C & D & $\mathbf{E}$ & $\mathbf{F}$ & G & H & I & $\mathbf{J}$ \\
\hline $\begin{array}{l}\text { Bactérias } \\
\text { aeróbias } \\
\text { mesófilas }\end{array}$ & $\begin{array}{l}1,3 \times 10^{4} \\
\text { UFC/g }\end{array}$ & $\begin{array}{l}1,0 \times 10^{5} \\
\text { UFC/g* }\end{array}$ & $\begin{array}{c}1,3 \times 10^{4} \\
\text { UFC/g } \\
\text { (est.) }\end{array}$ & $\begin{array}{c}1,0 \times 10^{4} \\
\text { UFC/g }\end{array}$ & $\begin{array}{c}5,3 \times 10^{2} \\
\text { UFC/g }\end{array}$ & $\begin{array}{c}5,2 \times 10^{2} \\
\text { UFC/g }\end{array}$ & $\begin{array}{c}4,3 \times 10^{2} \\
\text { UFC/g }\end{array}$ & $\begin{array}{c}3,8 \times 10^{2} \\
\text { UFC/g }\end{array}$ & $\begin{array}{c}1,0 \times 10^{2} \\
\text { UFC/g } \\
\text { (est.) }\end{array}$ & $\begin{array}{c}2,4 \times 10^{5} \\
\text { UFC/g }\end{array}$ \\
\hline $\begin{array}{l}\text { Estafilococus } \\
\text { coagulas e } \\
\text { positiva }\end{array}$ & $\begin{array}{l}4,5 \times 10^{2} \\
\text { UFC/g* }\end{array}$ & $\begin{array}{l}5,5 \times 10^{1} \\
\text { UFC/g* }\end{array}$ & $\begin{array}{l}7,3 \times 10^{2} \\
\text { UFC } / g^{\star}\end{array}$ & $\begin{array}{c}9,0 \times 10^{2} \\
\text { UFC/g } \\
\text { (est.) }\end{array}$ & $\begin{array}{l}6,0 \times 10^{2} \\
\text { UFC/g } \\
\text { (est.)* }^{*}\end{array}$ & $\begin{array}{c}<1,0 \times 10^{2} \\
\text { UFC/g } \\
\text { (est.) }\end{array}$ & $\begin{array}{c}2,2 \times 10^{2} \\
\text { UFC/g } \\
\text { (est.) }^{\star}\end{array}$ & $\begin{array}{c}1,0 \times 102 \\
\text { UFC/g } \\
\text { (est.) }\end{array}$ & $\begin{array}{c}3,0 \times 10^{2} \\
\text { UFC/g } \\
\text { (est.) }\end{array}$ & $\begin{array}{c}1,0 \times 10^{2} \\
\text { UFC/g } \\
\text { (est.) }\end{array}$ \\
\hline Bacillus cereus & $\begin{array}{c}3,0 \times 10^{2} \\
\text { UFC/g } \\
\text { (est.) }\end{array}$ & $\begin{array}{l}1,2 \times 10^{1} \\
\text { UFC/g } \\
\text { (est.)* }^{*}\end{array}$ & $\begin{array}{c}1,0 \times 10^{2} \\
\text { UFC/g } \\
\text { (est.) }\end{array}$ & $\begin{array}{c}\text { 4,0x10² } \\
\text { UFC/g } \\
\text { (est.) }\end{array}$ & $\begin{array}{l}\text { 8,3x10² } \\
\text { UFC/g } \\
\text { (est.)* }^{*}\end{array}$ & $\begin{array}{c}<1,0 \times 10^{2} \\
\text { UFC/g } \\
\text { (est.) }\end{array}$ & $\begin{array}{c}<1,0 \times 10^{2} \\
\text { UFC/g } \\
\text { (est.) }\end{array}$ & $\begin{array}{l}1,0 \times 10^{2} \\
\text { UFC/g }\end{array}$ & $\begin{array}{c}5,0 \times 10^{2} \\
\text { UFC/g }\end{array}$ & $\begin{array}{c}2,0 \times 10^{2} \\
\text { UFC/g }\end{array}$ \\
\hline Salmonella spp. & $\begin{array}{c}\text { Possível } \\
\text { presença } \\
\star\end{array}$ & $\begin{array}{c}\text { Possível } \\
\text { presença } \\
\text { * }\end{array}$ & $\begin{array}{c}\text { Possível } \\
\text { presença } \\
\star\end{array}$ & $\begin{array}{c}\text { Possível } \\
\text { presença } \\
\text { * }\end{array}$ & $\begin{array}{c}\text { Possível } \\
\text { presença } \\
\text { * }\end{array}$ & $\begin{array}{l}\text { Possível } \\
\text { presença } \\
\star\end{array}$ & $\begin{array}{c}\text { Possível } \\
\text { presença } \\
\star\end{array}$ & $\begin{array}{c}\text { Possível } \\
\text { presença } \\
\star\end{array}$ & $\begin{array}{c}\text { Possível } \\
\text { presença } \\
\star\end{array}$ & $\begin{array}{c}\text { Possível } \\
\text { presença } \\
\star\end{array}$ \\
\hline $\begin{array}{l}\text { Coliformes } \\
\text { totais }\end{array}$ & $\begin{array}{l}>1,1 \times 10^{3} \\
\mathrm{NMP} / \mathrm{g}^{\star}\end{array}$ & $\begin{array}{l}>1,1 \times 10^{3} \\
\mathrm{NMP} / \mathrm{g}^{\star}\end{array}$ & $\begin{array}{l}4,3 \times 10^{2} \\
\mathrm{NMP} / \mathrm{g}^{\star}\end{array}$ & $\begin{array}{l}>1,1 \times 10^{3} \\
\mathrm{NMP} / \mathrm{g}^{\star}\end{array}$ & $\begin{array}{l}9,3 \times 10^{2} \\
\mathrm{NMP} / \mathrm{g}^{*}\end{array}$ & $\begin{array}{l}2,3 \times 10^{2} \\
\mathrm{NMP} / \mathrm{g}^{\star}\end{array}$ & $\begin{array}{l}7,4 \times 10^{1} \\
\text { NMP/g }\end{array}$ & $\begin{array}{l}1,5 \times 10^{4} \\
\mathrm{NMP} / \mathrm{g}^{\star}\end{array}$ & $\begin{array}{l}2,1 \times 10^{2} \\
\mathrm{NMP} / \mathrm{g}^{\star}\end{array}$ & $\begin{array}{l}1,1 \times 10^{3} \\
\mathrm{NMP} / \mathrm{g}^{\star}\end{array}$ \\
\hline $\begin{array}{l}\text { Coliformes } \\
\text { termotolerantes }\end{array}$ & $\begin{array}{l}2,0 \times 10^{1} \\
\mathrm{NMP} / \mathrm{g}\end{array}$ & $\begin{array}{l}>1,1 \times 10^{5} \\
\mathrm{NMP} / \mathrm{g}^{\star}\end{array}$ & $\begin{array}{l}4,3 \times 10^{1} \\
\mathrm{NMP} / \mathrm{g}^{\star}\end{array}$ & $\begin{array}{l}1,1 \times 10^{1} \\
\text { NMP/g }\end{array}$ & $\begin{array}{l}9,3 \times 10^{2} \\
\text { NMP/g* }\end{array}$ & $\begin{array}{l}9,3 \times 10^{2} \\
\text { NMP/g* }\end{array}$ & $\begin{array}{l}<3,0 \\
\mathrm{NMP} / \mathrm{g}\end{array}$ & $\begin{array}{l}2,9 \times 10^{4} \\
\mathrm{NMP} / \mathrm{g}^{\star}\end{array}$ & $\begin{array}{l}3,5 \times 10^{1} \\
\mathrm{NMP} / \mathrm{g}^{\star}\end{array}$ & $\begin{array}{l}1,5 \times 10^{1} \\
\text { NMP/g* }\end{array}$ \\
\hline
\end{tabular}

${ }^{*}$ Amostra imprópria para consumo humano segundo padrão microbiológico estabelecido pela RDC $n^{0} 12$ da ANVISA (BRASIL, 2001).

${ }^{* *}$ Amostra imprópria para consumo humano segundo limite para Coliformes Termotolerantes da RDC nº12 da ANVISA (BRASIL, 2001).

*** Amostra imprópria para consumo humano segundo critérios de avaliação de Alves, Ueno (2010). 
Em estudo similar, Barcelos et al. (2016) constataram que, ao analisarem 20 amostras de saladas contendo maionese servidas em restaurantes de Ji-Paraná, Rondônia, Brasil, 30\% apresentaram contaminação por Salmonella spp.

Alves e Ueno (2010), ao avaliarem seis amostras de maionese de restaurantes self-service, obtiveram contagens de bactérias aeróbias mesófilas acima de $6,15 \times 10^{4} \mathrm{UFC} / \mathrm{g}$ em todas as amostras e contagem elevada de coliformes termotolerantes em uma amostra. No entanto, nenhuma amostra excedeu os limites microbiológicos para $S$. aureus e Salmonella spp.

Da mesma forma, as 15 amostras de saladas adicionadas de molho de maionese, provenientes de restaurantes self-service de Uberlândia, Minas Gerais, Brasil, apresentaram resultados dentro dos valores permitidos pela legislação para coliformes termotolerantes e de $S$. aureus (GUERRA; MIGUEL, 2011).

\section{CONCLUSÃO}

Das amostras de alimentos da cadeia quente e da cadeia fria, provenientes dos dez restaurantes participantes desta pesquisa, apenas três amostras de carnes e seis de macarrão apresentaram-se aptas ao consumo humano. As demais apresentaram contaminações acima dos limites da resolução $n^{\circ} 12 / 2001$, da ANVISA para, pelo menos, um microorganismo.

Os resultados obtidos para os alimentos da cadeia quente foram mais satisfatórios do que os da cadeia fria. Esse fato pode ser explicado pelo tratamento térmico a que os alimentos foram submetidos durante a cocção e que colaborou para a redução da carga microbiana.

Um fator que favorece 0 desenvolvimento microbiano e, consequentemente, a contaminação dos alimentos, é o emprego de temperaturas inadequadas durante a disposição dos alimentos aos clientes. Nos restaurantes avaliados, nenhuma amostra de alimento da cadeia fria estava em temperatura adequada, e apenas duas amostras de alimentos da cadeia quente estavam expostas na temperatura correta. Esta irregularidade é considerada crítica na oferta de alimento seguro, podendo comprometer a saúde dos consumidores.

Assim, esses resultados servem de alerta para as autoridades sanitárias, pelo risco potencial que esses alimentos contaminados possuem de causar DTAs aos consumidores.

\section{REFERÊNCIAS}

ABERC - Associação Brasileira de Refeições Coletivas. Mercado real. Disponível em: <http://www.aberc. com.br/mercadoreal.asp?IDMenu=21>. Acesso em: 21 fev. 2017.

AFIFI, H. S.; ABUSHELAIBI, A. A. Assessment of personal hygiene knowledge, and practices in Al Ain, United Arab Emirates. Food Control, v.25, n.1, p.249-253, 2012.

ALVES, M. G.; UENO, M. Restaurantes self-service: segurança e qualidade sanitária dos alimentos servidos.

Revista de Nutrição, Campinas, v.23, n.4, p.573-580, jul./ago. 2010.

ALVES, M.; MESQUITA, M. 0. de. Monitoramento da temperatura de preparações frias de uma unidade de alimentação e nutrição comercial da cidade de Santa Maria - RS. In: JORNADA INTERDISCIPLINAR EM SAÚDE, 3., Santa Maria, RS, 2010.

BADARÓ, A. C. L. Boas práticas para serviços de alimentação: um estudo em restaurantes comerciais do município de Ipatinga, Minas Gerais. 2007. $172 f$. Dissertação (Mestrado em Ciência da Nutrição) Universidade Federal de Viçosa, Viçosa, 2007.

BARCELOS, I. B. et al. Pesquisa de Salmonella spp. e Listeria monocytogenes em saladas contendo maionese comercializadas em restaurantes localizados no município de Ji-Paraná, Rondônia, Brasil. J Health Sci, v.18, n.3, p.159-162, 2016. 
BRAGA, A. C.; PEREIRA, T. L.; JUNIOR, P. P. A. Avaliação de restaurante universitário por meio de indicadores de qualidade. Editora Unijuí, v.13, n.30, p.306-326, abr./jun. 2015.

BRASIL. Ministério da Saúde. Resolução RDC n¹2 de 2 de janeiro de 2001. Regulamento técnico sobre padrões microbiológicos para alimentos. Diário Oficial da República Federativa do Brasil, Brasília, 2 de janeiro de 2001.

BRASIL. Ministério da Saúde. Resolução RDC n²16, de 15 de setembro de 2004. Regulamento técnico de boas práticas para serviços de alimentação. Diário Oficial da República Federativa do Brasil, Brasília, 16 de setembro de 2004.

CALIL, E. M. B. et al. Qualidade microbiológica de saladas oferecidas em restaurantes tipo self-service. Atas de Saúde Ambiental-ASA, v.1, n.1, p.36-42, 2013.

DOLINGER, E. J. O. et al. Contaminação microbiológica de alimentos comercializados em restaurantes de auto-serviço de Itumbiara-G0. Biotemas, v.24, n.4, p.129-133, 2010.

GUERRA, C. B.; MIGUEL, D. P. Staphylococcus coagulase positiva e coliformes fecais em pratos frios adicionados de molho de maionese. FAZU em Revista, Uberaba, n.8, p.131-136. 2011.

IBGE - Instituto Brasileiro de Geografia e Estatística. Pesquisa de orçamentos familiares 2008-2009: despesas, rendimentos e condições de vida. Rio de Janeiro, 2010. Disponível em: <http://biblioteca.ibge. gov.br/visualizacao/livros/liv45130.pdf>. Acesso em: 21 fev. 2017.

LIMA, E. N. S. et al. Análises microbiológicas de saladas e água servidas em um restaurante universitário do Triângulo Mineiro, Minas Gerais, Brasil. Enciclopédia Biosfera: Revista do Centro Científico Conhecer, Goiânia, v.11, n.22, p.3176, 2015.
LUES, J. F. R.; VANTONDER, I. The occurence of indicator bacteria on hands and aprons of food handlers in the delicatessen sections of a retail group. Food Control, v.18, n.4, p.326-332, 2007.

MENDES, R.A.; COELHO, A.I.M.; AZEREDO, R.M.C. Contaminação por Bacillus cereus em superfícies de equipamentos e utensílios em unidade de alimentação e nutrição. Ciência \& Saúde Coletiva, v.16, n.9, p.3933-3938, 2011.

OLIVEIRA, L. C. et al. Avaliação das temperaturas das preparações dos restaurantes self-service do hipercentro de Belo Horizonte/MG. HU Revista, Juiz de Fora, v.38, n.3/4, p.167-173, jul./dez. 2012.

PAIVA, J. F. F. Qualidade microbiológica das guarnições, saldas e molhos servidos em restaurantes universitários do DF. 2016. $22 f$. Monografia (graduação em Nutrição) - Universidade de Brasília, Brasília, 2016.

PENEDO, A. 0. et al. Avaliação das temperaturas dos alimentos durante 0 preparo e distribuição em restaurantes comerciais de Belo Horizonte - MG. Demetra, v.10, n.2, p.429-440, 2015.

RESTA, M. S. A.; OLIVEIRA, T. C. R. M. Avaliação do padrão estafilococos coagulase positiva estabelecido pela legislação brasileira para massas alimentícias. Food Technology. Campinas, SP, v.16, n.4, p.319325, 2013.

ROCHA,A. N. F.; SOARES, R. P.; BESERRA, M. L. S.Análise microbiológica de saladas cruas em restaurantes de Teresina - PI. Revista Interdisciplinar: Revista do Centro Universitário Uninovafapi, v.7, n.2, p.11-17, abr./mai./jun., 2014.

RODRIGUES, R. S. et al. Qualidade higienicossanitária das alfaces servidas em restaurantes self-service de Natal, RN. Higiene Alimentar, v.26, n.210/211, 2012.

SANTA, D. et al. Qualidade microbiológica de saladas disponibilizadas em restaurantes self-service. Higiene Alimentar, v.25, n.198/199, 2011. 
SANTOS, V. F. N.; BASSI, S. N. Avaliação da temperatura dos equipamentos e alimentos servidos em unidades de alimentação e nutrição na cidade de São Paulo. Linkania, v.5, n.1, p.110-125, 2015.

SÃO JOSÉ, J. F. B. Contaminação microbiológica em serviços de alimentação. Nutrire, São Paulo, SP, v.37, n.1, p.78-92, 2012.

SEBRAE. Serviço de Apoio às Micro e Pequenas Empresas de Santa Catarina. Santa Catarina em números: Canoinhas. Florianópolis, 2013. Disponível em:<http://www.sebrae.com.br/Sebrae/Portal\%20 Sebrae/UFs/SC/Anexos/canoinhas.pdf>. Acesso em: 21 fev. 2017.

SEREJO, M. T. T. et al. Análise da temperatura dos alimentos de restaurantes do tipo self-service em São Luís/MA. In: CONGRESSO DE PESQUISA E INOVAÇÃO DA REDE NORTE E NORDESTE DE EDUCAÇÃO TECNOLÓGICA, 4., 2010, Belém, PA, 2010.

SILVA, N. et al. Manual de métodos de análise microbiológica de alimentos e água. 4.ed. São Paulo: Varela, 2010.

Recebido em: 27 de fevereiro de 2017 Aceito em: 03 de maio de 2017 